\title{
EFFECT OF POSTNATAL COMPLICATIONS ON DEVELOPMENT AFTER MODERATE PRETERM BIRTH
}

J.M. Kerstjens ${ }^{1}$, I.F. Bocca-Tjeertes ${ }^{1}$, A.F. de Winter $^{2}$, G.B.J. Nijhuis ${ }^{3}$, J. Hornman ${ }^{3}$, S.A. Reijneveld ${ }^{2}$, A.F. Bos $^{1}$

${ }^{1}$ Neonatology, Beatrix Children's Hospital, University Medical Center Groningen, ${ }^{2}$ Social Sciences, University Medical Center Groningen, University of Groningen, ${ }^{3}$ Pediatrics, Beatrix Children's Hospital, University Medical Center Groningen, Groningen, The Netherlands

Background: Moderate preterms $\left(32-35^{+6}\right.$ weeks gestation) are at risk for developmental delay compared to fullterms. It is unknown if postnatal complications are associated with developmental delay for this group.

Objective: To determine the effect of postnatal complications after moderate preterm birth on development for moderate preterms at age 4 .

Design and methods: In a community-based stratified cohort, parents of 902 moderate preterms born in 2002/2003 completed the Ages and Stage Questionnaire (ASQ) when the child was 43-49 months old. An "ASQ total score" $>2$ SD below the reference value for fullterms was considered to indicate developmental delay. Postnatal risk factors including Agpar-score, transport after birth, tertiary NICU admission, documented asphyxia, mechanical ventilation, CPAP, inotropics, hypoglycemia $(<1.7 \mathrm{mmol} / \mathrm{ltr})$, proven or suspected sepsis, apneas, caffein, hyperbilirubinemia $(>255 \mathrm{mmol} / \mathrm{ltr})$, and gestational age at discharge were obtained retrospectively from medical records. We performed Chi-square analyses and constructed a multivariate logistic model starting with all postnatal factors with univariate association with developmental delay at $P<0.20$, adjusting for maternal education and SGA.

Results: Hypoglycemia (Odds ratio (OR) 2.5, Confidence Interval (CI); 1.2-5.0), documented asphyxia (OR 3.2, CI; 1.0-9.9) and tertiary NICU admission (OR $1.9 \mathrm{CI}$; 1.1-3.5) were positively associated with developmental delay. Gestational age at discharge had a borderline positive association with developmental delay $(P<0.20)$. All other postnatal risk factors were not associated with developmental delay $(P>0.2)$. In the multivariate model hypoglycemia remained associated with developmental delay (OR 2.6, CI; 1.3-5.3).

Conclusions: Hypoglycemia increases the odds for developmental delay after moderate preterm birth. 